\title{
Association between adenovirus viral load and mortality in pediatric allo-HCT recipients: the multinational AdVance study
}

\author{
Marco Zecca ${ }^{1}$ Robert Wynn ${ }^{2}$ - Jean-Hugues Dalle ${ }^{3}$ - Tobias Feuchtinger ${ }^{4}$ - Enrikas Vainorius ${ }^{5}$. \\ Thomas M. Brundage ${ }^{5}$. Aastha Chandak ${ }^{6}$. Essy Mozaffari ${ }^{5}$. Garrett Nichols ${ }^{5}$. Franco Locatelli ${ }^{7}$
}

Received: 20 December 2018 / Revised: 24 January 2019 / Accepted: 9 February 2019 / Published online: 25 February 2019

(c) The Author(s) 2019. This article is published with open access

\begin{abstract}
This multivariable analysis from the AdVance multicenter observational study assessed adenovirus (AdV) viremia peak, duration, and overall $\mathrm{AdV}$ viral burden — measured as time-averaged area under the viremia curve over 16 weeks $\left(\mathrm{AAUC}_{0-16}\right)$ - as predictors of all-cause mortality in pediatric allo-HCT recipients with AdV viremia. In the 6 months following allo-HCT, 241 patients had AdV viremia $\geq 1000$ copies $/ \mathrm{ml}$. Among these, 18\% (43/241) died within 6 months of first $\mathrm{AdV} \geq 1000$ copies $/ \mathrm{ml}$. Measures of AdV viral peak, duration, and overall burden of infection consistently correlate with all-cause mortality. In multivariable analyses, controlling for lymphocyte recovery, patients with $\mathrm{AdV} \mathrm{AAUC}_{0-16}$ in the highest quartile had a hazard ratio of 11.1 versus the lowest quartile (confidence interval 5.3-23.6); for peak AdV viremia, the hazard ratio was 2.2 for the highest versus lowest quartile. Both the peak level and duration of AdV viremia were correlated with short-term mortality, independent of other known risk factors for AdV-related mortality, such as lymphocyte recovery. AdV $\mathrm{AAUC}_{0-16}$, which assesses both peak and duration of $\mathrm{AdV}$ viremia, is highly correlated with mortality under the current standard of care. New therapeutic agents that decrease $\mathrm{AdV} \mathrm{AAUC}_{0-16}$ have the potential of reducing mortality in this at-risk patient population.
\end{abstract}

Supplementary information The online version of this article (https:// doi.org/10.1038/s41409-019-0483-7) contains supplementary material, which is available to authorized users.

Marco Zecca

m.zecca@smatteo.pv.it

1 Pediatric Hematology/Oncology, Fondazione IRCCS Policlinico S. Matteo, Pavia, Italy

2 Royal Manchester Children's Hospital, Manchester, UK

3 Robert Debré Hospital, Assistance Publique-Hôpitaux de Paris and Paris Diderot University, Paris, France

4 Department of Pediatric Hematology/Oncology and Stem Cell Transplantation, Dr. von Hauner University Children's Hospital LMU Munich, Munich, Germany

5 Chimerix, Durham, NC, USA

6 Analytica Laser, New York, NY, USA

7 Department of Pediatric Hematology/Oncology, IRCCS, Ospedale Pediatrico Bambino Gesù, University La Sapienza, Rome, Italy

\section{Introduction}

Pediatric allogeneic hematopoietic stem cell transplant (allo-HCT) recipients with nonmatched sibling sources are at high risk of developing adenovirus (AdV) infections, particularly during the immediate posttransplant period before immune reconstitution has occurred [1-7]. AdV viremia is widely considered to be an indicator of disease dissemination $[6,8,9]$, with higher levels of AdV viremia ( $\geq 1000$ copies $/ \mathrm{ml}$ ) shown to be associated with mortality in single-center studies [7, 10]. Guidelines have thus recommended antiviral treatment in high-risk patients with AdV viremia [11].

While there are few treatment options for AdV infection in allo-HCT recipients, the Infectious Diseases Working Party of the European Society of Blood and Marrow Transplantation recommends, whenever feasible, tapering immunosuppression and considering off-label treatment with intravenous (IV) cidofovir when AdV viremia is documented [7]. However, data supporting efficacy of IV cidofovir are limited, and treatment responses are generally observed only when immune reconstitution has occurred $[11,12]$. The use of IV cidofovir is also associated with 
nephrotoxicity, particularly in younger children [13]. Until a more effective or less toxic therapy is available, however, treatment with IV cidofovir is likely to be considered for allo-HCT recipients with confirmed AdV viremia.

Although there is accumulating evidence of an association between AdV viremia and mortality, these data come from small or single-center studies, thus raising questions as to how generalizable the findings may be for other centers, settings, or countries $[1,14,15]$. There is therefore a critical need for robust data from multinational and multicenter studies evaluating the relationship between AdV viral burden and mortality in pediatric allo-HCT recipients under the current standard of care in order to better define the baseline upon which therapeutic improvements may be built.

In order to understand the relationship between $\mathrm{AdV}$ viremia and outcomes, it is important to identify the viral dynamic parameters that best define the course of $\mathrm{AdV}$ viremia. It is known that the median time to AdV clearance with treatment with cidofovir is 9 weeks (range, 615 weeks) [12]. Previous studies have also shown that peak $\mathrm{AdV}$ viremia levels are independently associated with mortality; [16] the impact of persistent viremia, even at lower levels, could indicate ongoing organ damage for this lytic viral disease [17]. Other parameters, such as timeaveraged AdV area under the curve (AAUC), that incorporate both peak and persistence of $\mathrm{AdV}$ viremia could also correlate with clinical outcomes.

We investigated the association of $\mathrm{AdV}$ viral dynamics with all-cause mortality in pediatric patients with $\mathrm{AdV}$ viremia $\geq 1000$ copies/ml using data from the AdVance study, a multicenter retrospective study of AdV infection in allo-HCT recipients under current management $[11,18]$.

\section{Methods}

\section{Study design and participants}

AdVance was a retrospective, multicenter, multinational study investigating the incidence, management, and clinical outcomes of AdV infection in 2540 adult and 1736 pediatric allo-HCT recipients. There were 50 participating centers from seven European countries, including the United Kingdom, France, Germany, Italy, Spain, the Netherlands, and the Czech Republic. Of the 50 participating centers, at least 37 treated one or more pediatric patients. The study captured data from allogeneic transplants performed at the participating centers between January 2013 and September 2015, during a timeframe preceding the availability of brincidofovir at each center, and included follow-up data from all subjects until death or 1 year posttransplant. This analysis focuses on pediatric ( $<18$ years) allo-HCT recipients who had AdV viremia (measured either in plasma or whole blood) $\geq 1000$ copies $/ \mathrm{ml}$ within the first 6 months post-HCT.

Data were extracted by qualified and trained personnel at each study center from medical records and case report forms for all pediatric allo-HCT recipients who met inclusion criteria. The study was approved by institutional review boards/ethics committees at each participating center and data collection and use were in accordance with local and national laws. Extracted data included demographics, underlying disease for which allo-HCT was performed, details of the stem cell source used and type of donor, use of ex vivo T-cell depletion or serotherapy, and presence of comorbidities (Supplementary Table 1). Lowest lymphocyte count and highest serum creatinine level within 30-day windows were captured, as were highest organ stage of graft-versus-host disease (GvHD) at time of AdV viremia $\geq$ 1000 copies/ml and use of renal replacement (hemodialysis, peritoneal dialysis, ultrafiltration, and continuous venovenous filtration therapy). The results of all AdV tests performed were captured, as were antivirals used. The primary outcome measure was mortality within 6 months of first AdV viremia $\geq 1000$ copies/ml.

\section{Statistical methods}

Potential predictors of mortality were included in univariate and multivariable modeling. Factors with a $P$-value $\leq 0.20$ in univariate analysis were assessed in the multivariable models with backward selection used to build the final model with an exit criterion of $P$-value $\leq 0.10$. Separate multivariable models were analyzed, each including one of six AdV virologic measures (Fig. 1). Data were censored at date of last follow-up, or date of death, if these events occurred before 16 weeks from first detection of AdV viremia $\geq 1000$ copies/ml; separate models were generated for overall mortality and mortality that occurred in the absence of relapsed disease. The virologic measures evaluated were:

1. Time-averaged AUC for $\mathrm{AdV}$ viremia from first detection of $\mathrm{AdV}$ viremia $\geq 1000$ copies $/ \mathrm{ml}$ for 16 weeks (AAUC $0-16$ ).

2. Peak viremia: defined as the peak level of $\mathrm{AdV}$ viremia attained within 16 weeks from first detection of $\mathrm{AdV}$ viremia $\geq 1000$ copies/ml.

3. Days with viremia $<1000$ copies/ml within 16 weeks after first detection of AdV viremia $\geq 1000$ copies/ml.

4. Days with undetectable viremia within 16 weeks after first detection of $\mathrm{AdV}$ viremia $\geq 1000$ copies $/ \mathrm{ml}$.

5. Two-week change in AdV viremia: defined as change at 2 weeks from first detection of $\mathrm{AdV}$ viremia $\geq 1000$ copies/ml. This change (positive or negative) is calculated by subtracting AdV viremia at first detection $\geq 1000$ copies/ml from AdV viremia at 2 weeks. 


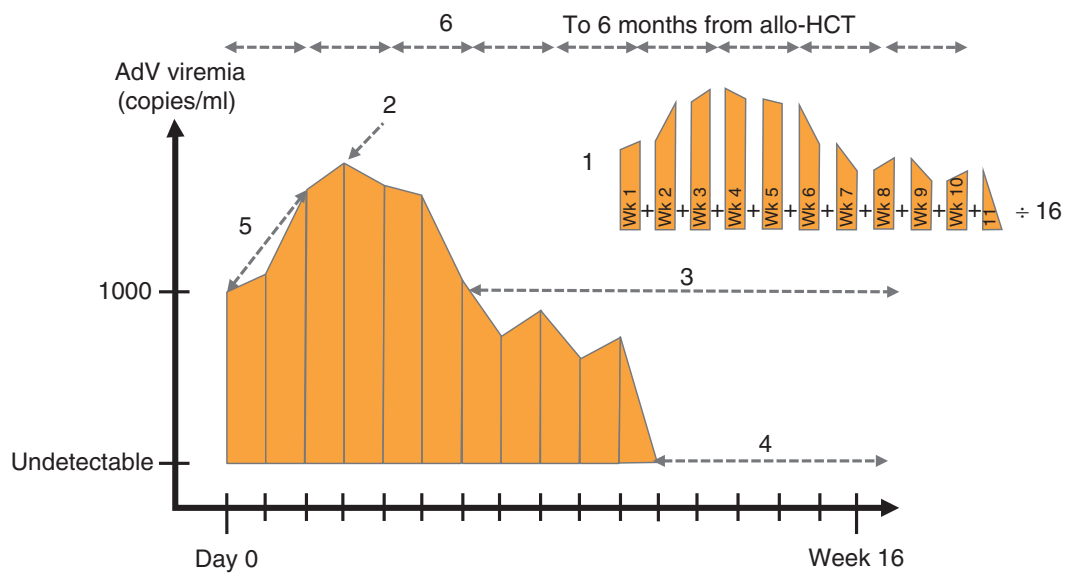

1. AdV AAUC $0-16$ :

$\log _{10}$ of the time-averaged area under the AdV viremia curve over the 16 weeks following first AdV viremia $\geq 1000$ copies $/ \mathrm{ml}$

2. Peak AdV viremia:

Peak $\log _{10}$ AdV viremia over the 16 weeks following first AdV viremia $\geq 1000$ copies $/ \mathrm{ml}$

3. Days with AdV viremia < 1000 copies $/ \mathrm{ml}$ :

Number of days where AdV viremia was $<1000$ copies $/ \mathrm{ml}$ over the 16 weeks following first $\mathrm{AdV}$ viremia $\geq 1000$ copies/ml

Fig. 1 AdV viral load dynamics

6. Change in AdV viremia over time: peak AdV viremia as a time-varying covariate for the hazard of mortality for each patient in 15-day windows from Day 0 to Day 180 or until death or date of last follow-up.

In addition to the virologic measures noted above, the following clinical and demographic factors were assessed as potential covariates in each of the models:

1. Immune reconstitution measured as total lymphocyte count categories of $<300,300-899, \geq 900$ cells $/ \mu l$ in 30-day time-dependent windows from Day 0 to Day 180 or until death or date of last follow-up.

2. Use of cidofovir, defined as time-varying covariate from the date of first use.

3. Maximum GvHD stage across skin, liver, and gastrointestinal tract at the time of first detection of AdV viremia $\geq 1000$ copies/ml (stage 0 , stage 1 or 2, stage 3 or 4 ).

4. Use of renal replacement therapy as time-varying covariate from the date of first use.

5. Other baseline factors included gender, underlying disease, age at the time of transplant, type of donor, Tcell depletion/serotherapy, comorbidities (Supplementary Table 1), diagnosis of AdV disease (possible/ probable, definitive, or disseminated as per study protocol definition), time from allo-HCT to AdV infection, time from $\mathrm{AdV}$ infection to $\mathrm{AdV}$ viremia $\geq$
4. Days with undetectable AdV viremia:

Number of days where AdV viremia was undetectable over the 16 weeks following first $\mathrm{AdV}$ viremia $\geq 1000$ copies $/ \mathrm{ml}$

5. 2-week change in AdV viremia:

Change in $\log _{10}$ AdV viremia in the first 2 weeks from first AdV viremia $\geq 1000$ copies $/ \mathrm{ml}$

6. Change in AdV viremia over time:

Peak $\log _{10}$ AdV viremia in 15-day time windows for 6 months after allo-HCT

1000 copies $/ \mathrm{ml}$, time from allo-HCT to $\mathrm{AdV}$ viremia $\geq 1000$ copies $/ \mathrm{ml}$, and presence of other double-stranded DNA (dsDNA) viral coinfections (cytomegalovirus, Epstein-Barr virus, or BK virus), as defined in Table 1.

Cox proportional hazards models were used to examine time to all-cause mortality within 6 months of first AdV viremia $\geq 1000$ copies/ml. Fine-Gray models were used to examine time to nonrelapse-related mortality within 6 months of first AdV viremia $\geq 1000$ copies $/ \mathrm{ml}$, with relapse considered as a competing risk.

Quartile analyses were conducted to examine the association between all the virologic measures (with the exception of time-varying AdV viremia), and time to allcause and nonrelapse-related mortality. Patients were divided into quartiles according to the values of each of the continuous virologic measures, and the median values in each quartile were identified. The hazard ratios of the virologic measures (when used as a continuous variable) obtained from the final multivariable models were then used to calculate the hazard ratios for the comparison between the median values of the highest quartile versus the lowest quartile.

A Kaplan-Meier curve was generated for the time from first detection of $\mathrm{AdV}$ viremia $\geq 1000$ copies $/ \mathrm{ml}$ to all-cause mortality. Patients were censored at 6 months or at the date of last contact (if lost to follow-up before 6 months); the 
Table 1 Variables included in multivariable analysis models

\begin{tabular}{ll}
\hline Prognostic factors & Definition \\
\hline Lymphocyte count over time & $<300,300-899, \geq 900$ \\
& $\begin{array}{l}30 \text {-day time-dependent windows post first detection of AdV } \\
\text { viremia } \geq 1000 \text { copies } / \mathrm{ml}\end{array}$ \\
& Time-dependent covariate from time of treatment initiation \\
CDV use over time & $0,1-2,3-4$ \\
Acute GvHD (maximum grading across & \\
organs) & Time-dependent covariate from time of treatment initiation \\
Use of renal replacement therapy over time & UK, Spain, France, Italy, Germany, Netherlands, Czech \\
Country & Republic \\
& Male, female \\
Gender & Malignant, nonmalignant immunodeficient, nonmalignant \\
Underlying disease & immunocompetent \\
& $<2,2-<12,12-<18$ years \\
Age at time of transplant & MRD or MUD, MMD, haploidentical or cord blood \\
Type of donor & None, Alemtuzumab (Campath), ATG, ex vivo T-cell \\
T-cell depletion/serotherapy & depletion \\
Nome, $\geq 1$
\end{tabular}

Malignant: acute lymphoblastic leukemia, acute myeloid leukemia, chronic myelogenous leukemia, myelodysplasia, non-Hodgkin lymphoma, other malignancy. Nonmalignant immunodeficient: autoimmune disease, congenital immunodeficiency, other congenital. Nonmalignant immunocompetent: aplastic anemia, sickle cell anemia, thalassemia

$A T G$ antithymocyte globulin, $B K V$ BK virus, $C D V$ cidofovir, $C M V$ cytomegalovirus, $E B V$ Epstein-Barr virus, $M M D$ mismatched donor, $M R D$ matched related donor, $M U D$ matched unrelated donor patients were stratified by quartiles of $\mathrm{AdV} \mathrm{AAUC}_{0-16}$. For nonrelapse-related mortality, a cumulative incidence curve for time from first detection of AdV viremia $\geq 1000$ copies/ $\mathrm{ml}$ to nonrelapse-related mortality was generated. Relapse was considered as a competing risk, and patients were censored at 6 months or at the date of last contact or at the date of relapse (if lost to follow-up or relapsed before 6 months).

\section{Results}

AdVance included 1736 pediatric allo-HCT recipients transplanted during the study period. Of these, 32\% (558/ 1736) had at least one positive AdV test within 6 months of allo-HCT and 23\% (395/1736) had AdV viremia. This analysis was based on the 241 pediatric allo-HCT recipients who had AdV viremia $\geq 1000$ copies/ml (Table 2). The median age was 5 years, with a range of less than 1 to 17 years. The majority of patients were male (66\%), and matched unrelated transplants (42\%) were the most frequent. Most patients (87\%) had undergone ex vivo T-cell depletion or received serotherapy (antithymocyte globulin [ATG] or alemtuzumab). At the time of first detection of AdV viremia $\geq 1000$ copies $/ \mathrm{ml}, 80 \%$ of patients had lymphocyte counts $<300$ cells $/ \mu l$. Concurrent dsDNA viral infections were relatively common, with $43 \%$ of patients having at least one additional dsDNA viral infection at the time of first AdV infection detection. In these pediatric alloHCT recipients who developed AdV viremia $\geq 1000$ copies/ $\mathrm{ml}$ within 6 months after transplant, all-cause mortality was $18 \%$ (43/241; 95\% confidence interval [CI] 13.2-22.4), and nonrelapse-related mortality was $15 \%(35 / 241 ; 95 \%$ CI 10.3-18.8).

\section{Univariate and multivariable analyses}

Each of the six AdV viral load measures was associated with 6-month all-cause mortality in univariate models (Table 3). Each $\log _{10}$ increase in peak AdV viral load was associated with a $47 \%$ increase in the hazard for mortality. The relationship between peak viral load and mortality was 
Table 2 Demographics and baseline characteristics

\begin{tabular}{ll}
\hline Baseline characteristics & Pediatric patients $(N=$ \\
$241)$
\end{tabular}

Age, years

Mean (s.d.)

6.3 (4.9)

Median (range)

$5.0(<1.0-17.0)$

Age stratification, years, $n(\%)$

$<2$

$61(25.3)$

2 to $<6$

63 (26.1)

6 to $<12$

72 (29.9)

12 to $<18$

45 (18.7)

Gender, $n(\%)$

Male

159 (66.0)

Female

$82(34.0)$

Stem cell source, $n(\%)$

\section{BM}

$116(48.1)$

PBSC

$92(38.2)$

33 (13.7)

Cord blood unit(s)

Donor type ${ }^{\mathrm{a}}, n(\%)$

Matched unrelated

$100(41.5)$

Haploidentical

49 (20.3)

Mismatched $^{\mathrm{b}}$

43 (17.8)

Cord blood unit(s)

35 (14.5)

33 (13.7)

Matched related

95 (39.4)

\section{ATG}

62 (25.7)

Alemtuzumab (Campath)

53 (22.0)

Ex vivo

31 (12.9)

None

Time from $A d V$ infection to $A d V \geq 1000$ copies $/ m l$, days

Mean (s.d.)

$15.2(24.5)$

Median (range)

$7(0.0-146.0)$

IQR

$0.0-17.0$

$<28$ days, $n(\%)$

$201(83.4)$

$\geq 28$ days, $n(\%)$

40 (16.6)

Time from allo-HCT to AdV $\geq 1000$ copies $/ \mathrm{ml}$, days

Mean (s.d.)

$40.4(38.8)$

Median (range)

$26.0(0.0-174.0)$

IQR

$13.0-56.0$

$<28$ days, $n(\%)$

$130(53.9)$

$\geq 28$ days, $n(\%)$

$111(46.1)$

Disease for which allo-HCT was conducted, $n(\%)$

Malignant

148 (61.4)

Nonmalignant immunodeficient

72 (29.9)

Nonmalignant immunocompetent

21 (8.7)

dsDNA viral coinfections, $n(\%)$

None

138 (57.3)

$\geq 1$ dsDNA viral infection in addition to $\mathrm{AdV}$
Table 2 (continued)

\begin{tabular}{lc}
\hline Baseline characteristics & $\begin{array}{l}\text { Pediatric patients }(N= \\
241)\end{array}$ \\
\hline Comorbidities $^{\mathrm{d}}, n(\%)$ & $147(61.0)$ \\
None & $94(39.0)$ \\
$\geq 1$ & $14(5.8)$ \\
Lymphocyte count at time of AdV viremia $\geq$ & 1000 copies $/ \mathrm{ml}, n(\%)$ \\
$\geq 900$ & $35(14.5)$ \\
$300-899$ & $192(79.7)$ \\
$<300$ &
\end{tabular}

Maximum stage GvHD among skin, liver, GI at the time of $A d V$ viremia $\geq 1000$ copies $/ \mathrm{ml}, n(\%)$

$\begin{array}{ll}0 & 174(72.2) \\ 1 & 22(9.1) \\ 2 & 17(7.1) \\ 3 & 16(6.6) \\ 4 & 12(5.0)\end{array}$

ATG antithymocyte globulin, $B M$ bone marrow, $G I$ gastrointestinal, $I Q R$ interquartile range, $P B S C$ peripheral blood stem cell, s.d. standard deviation

${ }^{\mathrm{a} C}$ Categories are not mutually exclusive

${ }^{\mathrm{b}}$ Mismatching was determined according to standard practice at each study site

${ }^{\mathrm{c}} \mathrm{A}$ hierarchy was applied such that patients who received ex vivo Tcell depletion and ATG and/or alemtuzumab were counted in the ex vivo category

${ }^{\mathrm{d}}$ Listed in Supplementary Table 1

greatest at the top of the range- $10 \%$ of pediatric patients achieved peak AdV viral loads greater than $4.75 \times 10^{6}$ copies $/ \mathrm{ml}$, with correlating mortality of $58 \%$ (14/24 subjects); this was dramatically higher than the 13\% (29/ 217 subjects) mortality observed in the remaining $90 \%$ of the AdVance pediatric population. For $\mathrm{AdV} \mathrm{AAUC}_{0-16}$, each $\log _{10}$ increase in average viral burden was associated with a doubling of the hazard for mortality. Each day with AdV viremia $<1000$ copies/ml and each day with AdV undetectable over the 16 weeks after AdV viremia $\geq 1000$ copies/ml was associated with a $4 \%$ reduction in the hazard for mortality. In addition to the AdV viral load measures, the presence of AdV disease was also significantly associated with mortality. Time-updated lymphocyte counts, the use of IV cidofovir, maximum GvHD stage $\geq 3$, the use of renal replacement therapy, the presence of comorbidities, and donor type were associated with increased mortality risk (Table 3).

In multivariable analysis, each of the aforementioned virologic measures was a strong predictor of mortality in separate models (Fig. 2). In addition, time-updated low lymphocyte counts $(<300$ cells $/ \mu l)$, female gender, and use of renal replacement therapy were also significant predictors (Fig. 2). In the model including maximum viremia over 
Table 3 Univariate analysis

\begin{tabular}{|c|c|c|c|c|c|}
\hline Prognostic factor & Definition & Avg. $N$ & HR $(95 \%$ CI $)$ & Pairwise $P$-value & Overall $P$-value \\
\hline AdV AAUC $_{0-16}$ & Continuous & 241 & $1.99(1.69-2.35)$ & & $<0.0001$ \\
\hline Peak AdV viremia ${ }_{0-16}$ & Continuous & 241 & $1.47(1.29-1.67)$ & & $<0.0001$ \\
\hline Days $<1000$ copies $/ \mathrm{ml}_{0-16}$ & Continuous & 241 & $0.96(0.95-0.97)$ & & $<0.0001$ \\
\hline Days with undetectable viremia $a_{0-16 \mathrm{w}}$ & Continuous & 241 & $0.96(0.95-0.97)$ & & $<0.0001$ \\
\hline 2-week change in AdV viremia & Continuous & 241 & $1.35(1.15-1.59)$ & & 0.0003 \\
\hline Change in AdV viremia over time & Continuous & 241 & $1.54(1.39-1.71)$ & & $<0.0001$ \\
\hline \multirow[t]{3}{*}{ Lymphocyte counts (t) } & $\geq 900$ & 65.0 & & & $<0.0001$ \\
\hline & $300-899$ & 76.0 & $1.91(0.48-7.56)$ & 0.3557 & \\
\hline & $<300$ & 77.3 & $10.95(3.18-37.71)$ & 0.0001 & \\
\hline \multirow[t]{2}{*}{ CDV (t) use } & No & 121 & 1.00 & & 0.0310 \\
\hline & Yes & 120 & $1.98(1.06-3.68)$ & & \\
\hline \multirow[t]{3}{*}{ Maximum GvHD stage } & 0 & 174 & 1.00 & & 0.0153 \\
\hline & 1,2 & 39 & $0.76(0.29-1.97)$ & 0.5738 & \\
\hline & 3,4 & 28 & $2.67(1.30-5.51)$ & 0.0076 & \\
\hline \multirow[t]{2}{*}{ Renal replacement (t) } & No & 229 & 1.00 & & $<0.0001$ \\
\hline & Yes & 12 & $28.41(13.02-61.98)$ & & \\
\hline \multirow[t]{2}{*}{ Gender } & Male & 159 & 1.00 & & 0.1170 \\
\hline & Female & 82 & $1.62(0.89-2.95)$ & & \\
\hline \multirow[t]{2}{*}{ Type of donor } & MRD or MUD & 124 & 1.00 & & 0.0221 \\
\hline & MMD/Hapl/CBU & 117 & $2.08(1.11-3.89)$ & & \\
\hline \multirow[t]{2}{*}{ Comorbidities $^{\mathrm{a}}$} & None & 147 & 1.00 & & 0.0132 \\
\hline & $\geq 1$ & 94 & $2.14(1.17-3.91)$ & & \\
\hline \multirow[t]{2}{*}{ AdV disease } & No & 158 & 1.00 & & $<0.0001$ \\
\hline & Yes & 83 & $2.94(1.60-5.39)$ & & \\
\hline
\end{tabular}

$C B U$ cord blood (units), $C D V$ cidofovir, $H a p l$ haploidentical, $H R$ hazard ratio, $M M D$ mismatched donor, $M R D$ matched related donor, $M U D$ matched unrelated donor

${ }^{a}$ Listed in Supplementary Table 1

time, GvHD stage 3 or 4 was also a significant predictor of mortality (Fig. 2).

\section{Quartile analyses}

Analysis of virologic parameters by quartiles revealed that increasing viral load was consistently associated with increased all-cause mortality risk (Fig. 3). Fewer days with AdV viremia $<1000$ copies/ml or days with undetectable AdV viremia showed a strong association with higher mortality risk. For the comparison of the quartile with the fewest days with viremia $<1000$ copies $/ m$ l (Q1, $\leq 42$ days) versus the greatest number of days (Q4, $\geq 105$ days), the hazard ratio was 72.7 (95\% CI 24.5218.3). For undetectable viremia, the hazard ratio was 72.7 for patients with $\leq 21$ days (Q1) of undetectable viremia versus those with $\geq 98$ days (Q4) of undetectable viremia. AdV AAUC $\mathrm{A}_{0-16}$ was also associated with allcause mortality - patients in the highest quartile of $\mathrm{AdV}$ viral burden had an 11-fold increase in the hazard for mortality when compared with the lowest quartile $(95 \%$ CI 5.3-23.6) (Fig. 3).

Of the children who died, 79\% (34/43) had persistent $\mathrm{AdV}$ viremia at the last measurement. In those with $\mathrm{AdV}$ AAUC $_{0-16}$ in the top quartile, 97\% (30/31) of the patients who died still had $\mathrm{AdV}$ viremia at the last measurement. Corresponding analysis of nonrelapse-related mortality led to similar findings (Supplementary Figure 1).

Analysis of individual AdV viral load plots over time shows that peak and persistence of $\mathrm{AdV}$ viremia were far greater in the highest $\mathrm{AdV} \mathrm{AAUC}_{0-16}$ quartile compared with the lowest quartile (Fig. 4). All-cause mortality was significantly greater in the highest quartile versus the lowest quartile $(52 \%, 31 / 60$ vs 3\%, 2/60) (Fig. 5a), and the differences between quartiles were statistically significant $(P<$ 0.0001 for all quartile comparisons from chi-square test).

Nonrelapse-related mortality was also greatest in the highest AdV $\mathrm{AAUC}_{0-16}$ quartile $(43 \%, 26 / 60)$ compared with the lowest $\mathrm{AdV} \mathrm{AAUC}_{0-16}$ quartile $(0 \%, 0 / 60)$ (Fig. 5b). Differences between quartiles of AdV AAUC $\mathrm{A}_{0-16}$ 


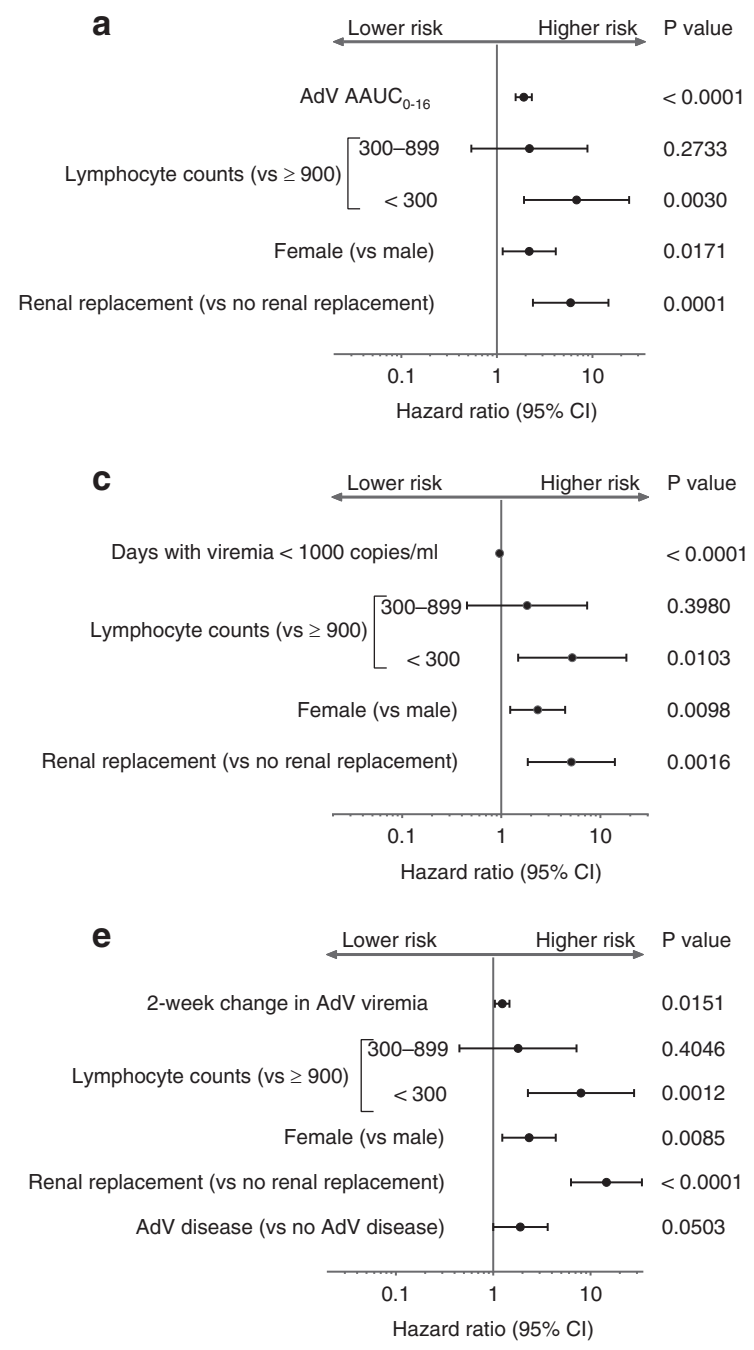

Fig. 2 All six measures of AdV viral load dynamics were independently associated with increased risk of all-cause mortality. a AdV $\mathrm{AAUC}_{0-16}$. b Peak AdV viremia ${ }_{0-16}$. c Days with viremia $<1000$

were statistically significant $(P<0.0001$ for all comparisons from chi-square test).

\section{Discussion}

AdVance is the first large retrospective multinational/multicenter study to examine the impact of AdV viremia on clinical outcomes in pediatric allo-HCT recipients. Whereas previous analyses have been from small or single-center studies $[1,14,15]$, AdVance is a broad representative study incorporating data from 50 centers in seven countries across Europe, at least 37 of which treated one or more pediatric patients.

Different measures can be used to assess viral dynamics: peak viral load, the duration of infection, and the change in viral load over time. For cytolytic viral infections such as $\mathrm{AdV}$, plasma viremia may provide a quantitative measure of

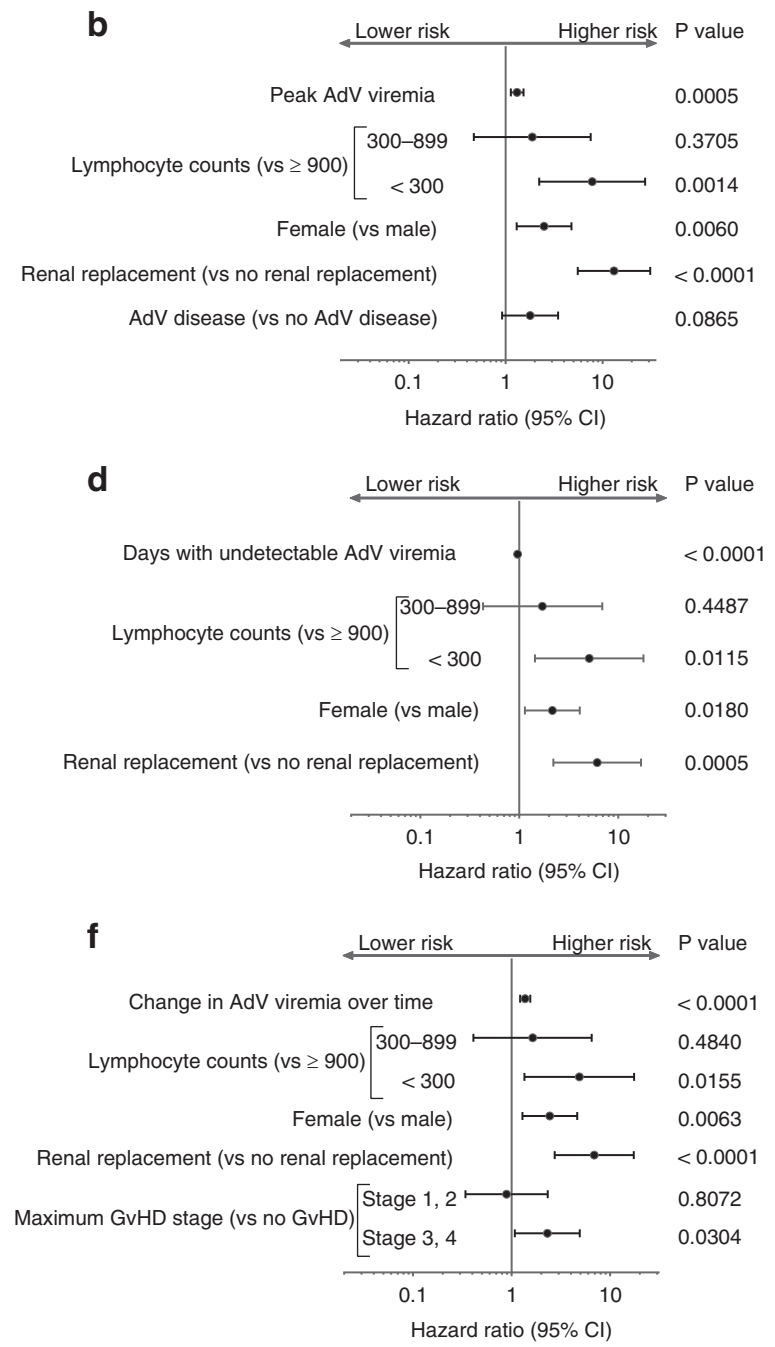

copies $/ \mathrm{ml}_{0-16}$. d Days with undetectable viremia $<1000$ copies $/ \mathrm{ml}_{0-16}$. e 2-week change in $\mathrm{AdV}$ viremia. $\mathbf{f}$ Change in $\mathrm{AdV}$ viremia over time

tissue damage in the most commonly affected organs: the gastrointestinal tract, lungs, liver, or kidneys [17]. In our study, over half the pediatric allo-HCT recipients who developed AdV viremia surpassed the level of $\geq 1000$ copies $/ \mathrm{ml}$, an accepted threshold for the initiation of preemptive treatment [7, 18]. Analyses of AdV viral load in these patients revealed a strong association with risk of mortality. Findings were similar for overall mortality and for nonrelapse-related mortality.

A lower number of days with undetectable AdV viremia or AdV viremia below the threshold of 1000 copies $/ \mathrm{ml}$ (i.e., more prolonged $\mathrm{AdV}$ viremia) was a strong predictor of mortality. AdV viral burden, assessed by AdV AAUC ${ }_{0-16}$, was strongly associated with mortality, and pediatric allo$\mathrm{HCT}$ recipients with the greatest viral burden (i.e., highest $\mathrm{AdV} \mathrm{AAUC}_{0-16}$ quartile) were at substantially greater risk of death than those with the lowest viral burden (ie, lowest $\mathrm{AdV} \mathrm{AAUC}_{0-16}$ quartile). 


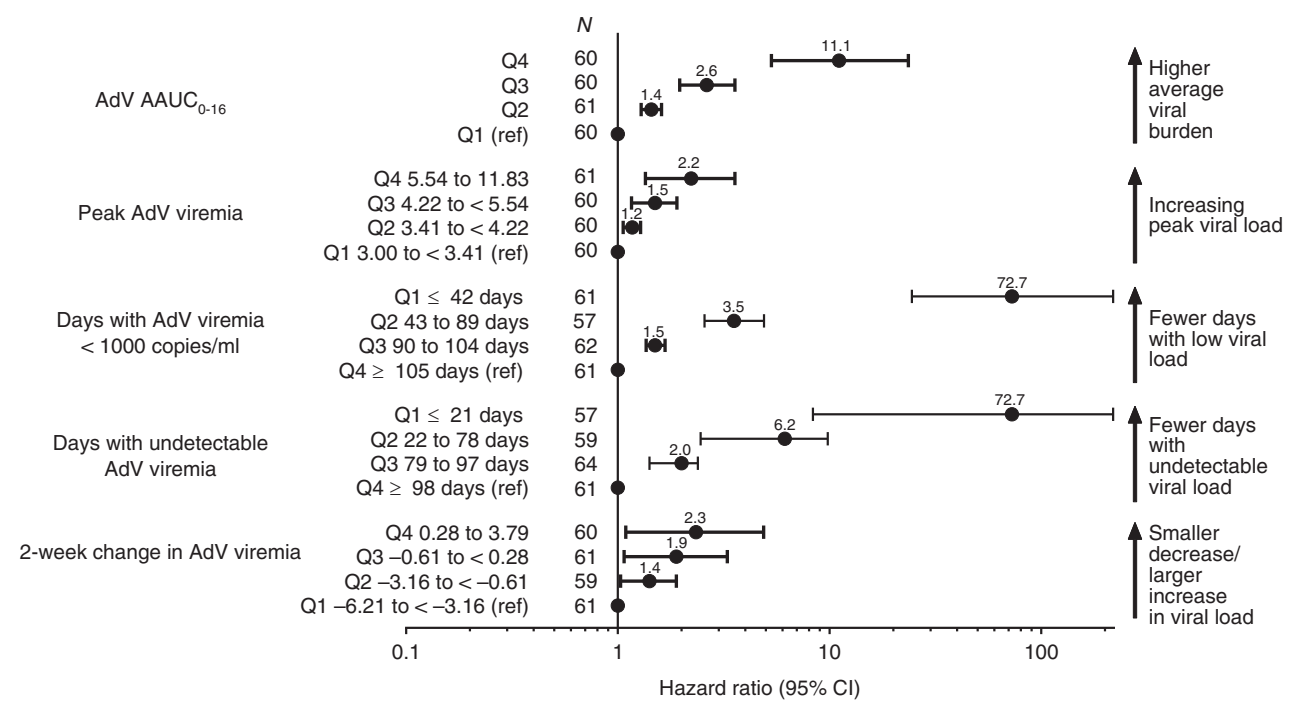

Fig. 3 Higher AdV viral load and burden were associated with a significantly greater risk of all-cause mortality in multivariable models. AdV AAUC ${ }_{0-16}, \log _{10}$ of the time-averaged area under the AdV viremia curve over the 16 weeks following first AdV viremia $\geq 1000$ copies/ml; Peak AdV viremia, peak $\log _{10}$ AdV viremia over the 16 weeks following first AdV viremia $\geq 1000$ copies/ml; Days with $\mathrm{AdV}$ viremia $<1000$ copies/ml, number of days where AdV viremia was $<1000$ copies/ml over the 16 weeks following first AdV viremia $\geq$ 1000 copies/ml; Days with undetectable AdV viremia, number of days where AdV viremia was undetectable over the 16 weeks following first AdV viremia $\geq 1000$ copies $/ \mathrm{ml}$; 2-week change in AdV viremia, change in $\log _{10}$ AdV viremia in the first 2 weeks from first AdV viremia $\geq 1000$ copies/ml. Q, quartile; ref, reference group. Note: Factors with $P \leq 0.20$ in the univariate analysis were brought forward into the multivariable models, followed by a backward selection to keep the factors with $P \leq 0.10$ in the final models. Lymphocyte count, gender, and renal replacement therapy were also significant $(P<0.05)$ prognostic factors in each of the final multivariable models

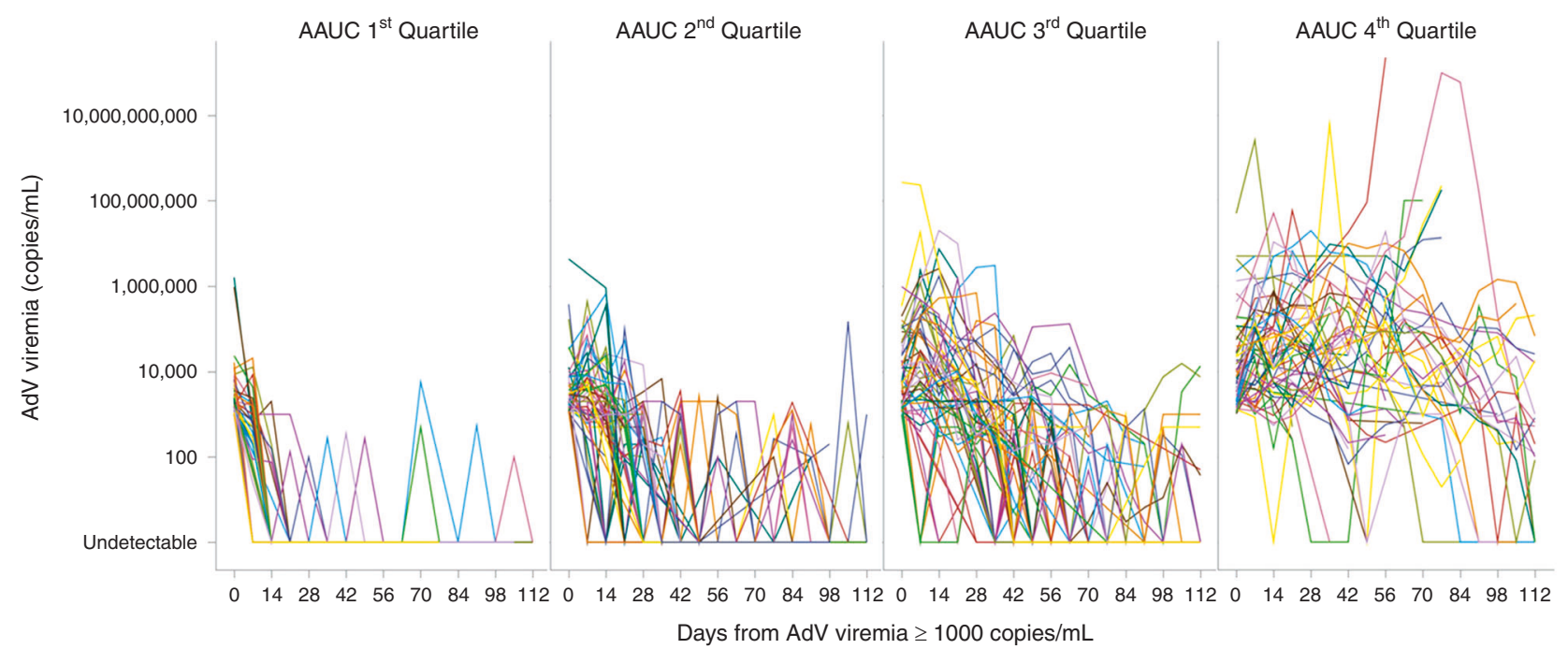

Fig. 4 Both AdV peak and persistence contribute to total AdV viral burden (as measured by AdV AAUC)

In separate multivariable models, all six AdV virologic measures were found to be an independent risk factor for mortality, along with previously identified risk factors for AdV-associated mortality, such as lymphocyte recovery or GvHD [19-21]. There was a high degree of concordance between the multivariable models, suggesting that both peak viremia and duration of infection were correlated with mortality.
Although competing risks for nonrelapse-related mortality (such as regimen-related toxicity, other infections, and GvHD) complicate attribution of cause of death to any one factor, the close temporal relationship of AdV viremia with death and the correlation of high viral burdens with mortality strongly suggest that AdV infection was a causative factor in most of these cases. Most of these deaths occurred within 2 months of first detection of AdV viremia $\geq 1000$ 
Fig. 5 Higher AdV viral burden is associated with lower probability of 6-month overall survival. a Kaplan-Meier plot of all-cause mortality. b Cumulative incidence plot of nonrelapse-related mortality
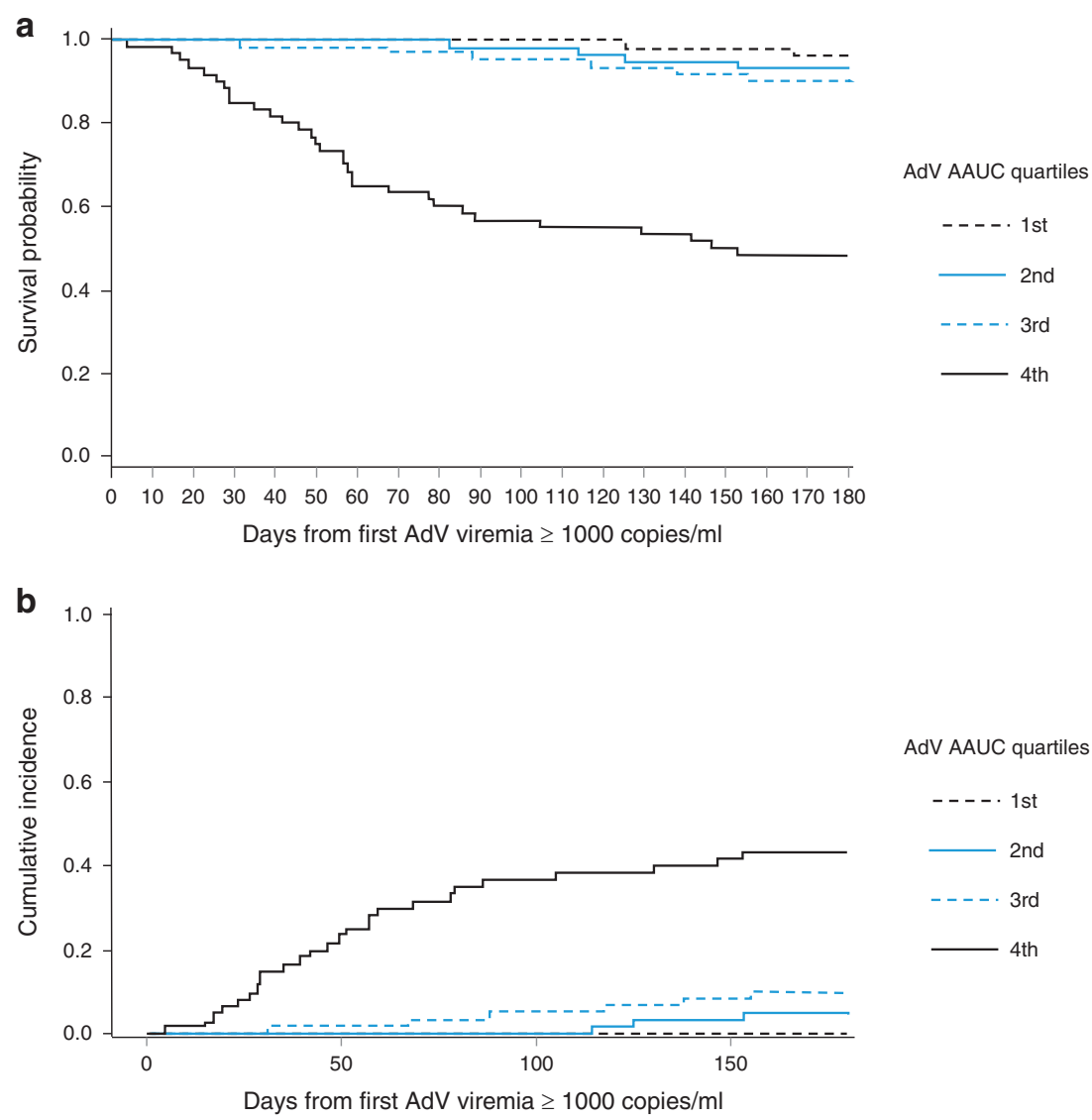

copies/ml despite receiving standard of care; the vast majority of subjects who died still had AdV viremia at the time of death, also suggesting that $\mathrm{AdV}$ was likely causal in many of these cases.

While measures of viral dynamics - such as peak AdV viremia, change in viral load over time, or days with undetectable AdV viremia-are clinically relevant measures of $\mathrm{AdV}$ viremia, AdV AAUC appeared to be a sensitive measure of the total viral burden experienced by the patient, incorporating both the peak and persistence of $\mathrm{AdV}$ in the plasma. AUC has been used to assess the efficacy of antivirals against influenza $\mathrm{A}$ and respiratory syncytial virus in clinical trials $[22,23]$. This parameter has also been used to assess the efficacy of treatments for HIV-1 [24]. Timeaveraged AUC extends upon this measure by accounting for variability in follow-up due to the competing mortality risk.

The failure to respond to standard-of-care antiviral therapy was associated with increased mortality in this study. Our findings suggest that prompt and sustained control of $\mathrm{AdV}$ viremia with more potent antivirals is likely to have a substantial impact on mortality in this patient population.

In line with all observational studies, the findings from AdVance are subject to limitations due to the retrospective nature of data capture and the potential for bias that cannot be fully accounted for in the statistical models. There was no central laboratory or centrally driven treatment approach, and thus the differences in the threshold for initiating antiviral therapy and/or the $\mathrm{AdV}$ polymerase chain reaction assay from institution to institution reduces precision. Notably, however, a survey of practice patterns at the centers participating in the AdVance study showed that monitoring and treatment practices were broadly similar and in line with the guidelines of the European Conference on Infections in Leukemia $[11,18]$.

Currently there are no approved treatment options for $\mathrm{AdV}$ viremia in allo-HCT recipients, and IV cidofovir is commonly used off-label [25]. While IV cidofovir can help stabilize AdV viremia during the period preceding immune reconstitution, AdV clearance by cidofovir generally requires support from cellular immunity, and nephrotoxicity remains a substantial drug-related side effect limiting its use [13, 15, 16, 26, 27]. Clearly, there is a need for more effective and less toxic treatment options for these patients to improve clinical outcomes.

In summary, the AdVance study represents the largest and most robust evaluation of the risk for mortality associated with AdV viremia in pediatric allo-HCT recipients. These results are based on an observational 'real-world' clinical course of $\mathrm{AdV}$ infection in pediatric allo-HCT recipients under standard of care. Several measures of AdV 
viral load were strongly and independently associated with mortality after pediatric allo-HCT_-both the peak and persistence of AdV viremia contribute to mortality risk. AdV $\mathrm{AAUC}_{0-16}$ incorporates both measures, with each $\log _{10}$ increase in $\mathrm{AdV}$ AAUC $\mathrm{A}_{0-16}$ associated with a near-doubling of mortality risk; these data suggest that interventions able to reduce AdV viral burden should decrease mortality. Our findings show that $\mathrm{AdV}$ viral dynamic measures including AdV AAUC $0-16$ are useful indicators of outcome in pediatric allo-HCT recipients with AdV viremia. These markers may be usefully employed in clinical trials aimed at validating the efficacy of novel therapies that have the potential to reduce the risk of mortality in these patients.

Acknowledgements The authors would like to thank the patients and investigators at each of the study sites. Medical writing and editorial support in the preparation of this manuscript was provided by Paul Hassan of Engage Scientific (Horsham, UK) and was funded by Chimerix Inc.

\section{Compliance with ethical standards}

Conflict of interest MZ, RW, JHD, TF, and FL are investigators on the AdVance study sponsored by Chimerix. EV, TMB, EM, and GN are employees of the study sponsor, Chimerix. AC is an employee of Analytica Laser who provided statistical support for the AdVance study.

Publisher's note: Springer Nature remains neutral with regard to jurisdictional claims in published maps and institutional affiliations.

Open Access This article is licensed under a Creative Commons Attribution 4.0 International License, which permits use, sharing, adaptation, distribution and reproduction in any medium or format, as long as you give appropriate credit to the original author(s) and the source, provide a link to the Creative Commons license, and indicate if changes were made. The images or other third party material in this article are included in the article's Creative Commons license, unless indicated otherwise in a credit line to the material. If material is not included in the article's Creative Commons license and your intended use is not permitted by statutory regulation or exceeds the permitted use, you will need to obtain permission directly from the copyright holder. To view a copy of this license, visit http://creativecommons. org/licenses/by/4.0/.

\section{References}

1. Lion T. Adenovirus infections in immunocompetent and immunocompromised patients. Clin Microbiol Rev. 2014;27:441-62. https://doi.org/10.1128/CMR.00116-13.

2. van Tol MJ, Claas EC, Heemskerk B, Veltrop-Duits LA, de Brouwer CS, van Vreeswijk T, et al. Adenovirus infection in children after allogeneic stem cell transplantation: diagnosis, treatment and immunity. Bone Marrow Transplant. 2005;35(Suppl 1):S73-76. https://doi.org/10.1038/sj.bmt.1704852.

3. Wasserman R, August CS, Plotkin SA. Viral infections in pediatric bone marrow transplant patients. Pediatr Infect Dis J. 1988;7:109-15.

4. Flomenberg P, Babbitt J, Drobyski WR, Ash RC, Carrigan DR, Sedmak GV, et al. Increasing incidence of adenovirus disease in bone marrow transplant recipients. J Infect Dis. 1994;169: $775-81$.

5. Howard DS, Phillips IG, Reece DE, Munn RK, Henslee-Downey J, Pittard M, et al. Adenovirus infections in hematopoietic stem cell transplant recipients. Clin Infect Dis. 1999;29:1494-501. https://doi.org/10.1086/313514.

6. Lion T, Baumgartinger R, Watzinger F, Matthes-Martin S, Suda M, Preuner S, et al. Molecular monitoring of adenovirus in peripheral blood after allogeneic bone marrow transplantation permits early diagnosis of disseminated disease. Blood. 2003;102:1114-20. https://doi.org/10.1182/blood-2002-07-2152.

7. Hiwarkar P, Kosulin K, Cesaro S, Mikulska M, Styczynski J, Wynn R, et al. Management of adenovirus infection in patients after haematopoietic stem cell transplantation: state-of-the-art and real-life current approach: a position statement on behalf of the Infectious Diseases Working Party of the European Society of Blood and Marrow Transplantation. Rev Med Virol. 2018;28: e1980 https://doi.org/10.1002/rmv.1980.

8. Chakrabarti S, Mautner V, Osman H, Collingham KE, Fegan CD, Klapper PE, et al. Adenovirus infections following allogeneic stem cell transplantation: incidence and outcome in relation to graft manipulation, immunosuppression, and immune recovery. Blood. 2002;100:1619-27. https://doi.org/10.1182/blood-2002-02-0377.

9. Sandkovsky U, Vargas L, Florescu DF. Adenovirus: current epidemiology and emerging approaches to prevention and treatment. Curr Infect Dis Rep. 2014;16:416 https://doi.org/10.1007/s11908014-0416-y.

10. Schilham MW, Claas EC, van Zaane W, Heemskerk B, Vossen $\mathrm{JM}$, Lankester AC, et al. High levels of adenovirus DNA in serum correlate with fatal outcome of adenovirus infection in children after allogeneic stem-cell transplantation. Clin Infect Dis. 2002;35:526-32. https://doi.org/10.1086/341770.

11. Matthes-Martin S, Feuchtinger T, Shaw PJ, Engelhard D, Hirsch $\mathrm{HH}$, Cordonnier $\mathrm{C}$, et al. European guidelines for diagnosis and treatment of adenovirus infection in leukemia and stem cell transplantation: summary of ECIL-4 (2011). Transpl Infect Dis. 2012;14:555-63. https://doi.org/10.1111/tid.12022.

12. Hiwarkar P, Amrolia P, Sivaprakasam P, Lum SH, Doss H, O'Rafferty C, et al. Brincidofovir is highly efficacious in controlling adenoviremia in pediatric recipients of hematopoietic cell transplant. Blood. 2017;129:2033-7. https://doi.org/10.1182/ blood-2016-11-749721.

13. Vora SB, Brothers AW, Englund JA. Renal toxicity in pediatric patients receiving cidofovir for the treatment of adenovirus infection. J Pediatr Infect Dis Soc. 2017;6:399-402. https://doi. org/10.1093/jpids/pix011.

14. La Rosa AM, Champlin RE, Mirza N, Gajewski J, Giralt S, Rolston $\mathrm{KV}$, et al. Adenovirus infections in adult recipients of blood and marrow transplants. Clin Infect Dis. 2001;32:871-6. https://doi.org/10.1086/319352.

15. Mynarek M, Ganzenmueller T, Mueller-Heine A, Mielke C, Gonnermann A, Beier R, et al. Patient, virus, and treatment-related risk factors in pediatric adenovirus infection after stem cell transplantation: results of a routine monitoring program. Biol Blood Marrow Transplant. 2014;20:250-6. https://doi.org/10. 1016/j.bbmt.2013.11.009.

16. Lugthart G, Oomen MA, Jol-van der Zijde CM, Ball LM, Bresters D, Kollen WJ, et al. The effect of cidofovir on adenovirus plasma DNA levels in stem cell transplantation recipients without $T$ cell reconstitution. Biol Blood Marrow Transplant. 2015;21:293-9. https://doi.org/10.1016/j.bbmt.2014.10.012.

17. Heim A. Advances in the management of disseminated adenovirus disease in stem cell transplant recipients: impact of adenovirus load (DNAemia) testing. Expert Rev Anti Infect Ther. 2011;9:943-5. https://doi.org/10.1586/eri.11.113. 
18. González-Vicent M, Verna M, Pochon C, Chandak A, Vainorius $\mathrm{E}$, Brundage $\mathrm{T}$, et al. Current practices in the management of adenovirus infection in allogeneic hematopoietic stem cell transplant recipients in Europe: The AdVance study. Eur J Haematol. 2019;102:210-7. https://doi.org/10.1111/ejh.13194.

19. Baldwin A, Kingman H, Darville M, Foot AB, Grier D, Cornish JM, et al. Outcome and clinical course of 100 patients with adenovirus infection following bone marrow transplantation. Bone Marrow Transplant. 2000;26:1333-8. https://doi.org/10.1038/sj. bmt.1702716.

20. Lee YJ, Chung D, Xiao K, Papadopoulos EB, Barker JN, Small $\mathrm{TN}$, et al. Adenovirus viremia and disease: comparison of T celldepleted and conventional hematopoietic stem cell transplantation recipients from a single institution. Biol Blood Marrow Transplant. 2013;19:387-92. https://doi.org/10.1016/j.bbmt.2012.10.014.

21. Shields AF, Hackman RC, Fife KH, Corey L, Meyers JD. Adenovirus infections in patients undergoing bone-marrow transplantation. N Engl J Med. 1985;312:529-33. https://doi.org/10. 1056/NEJM198502283120901.

22. DeVincenzo JP, Whitley RJ, Mackman RL, Scaglioni-Weinlich C, Harrison L, Farrell E, et al. Oral GS-5806 activity in a respiratory syncytial virus challenge study. N Engl J Med. 2014;371:711-22. https://doi.org/10.1056/NEJMoa1401184.

23. Vegvari C, Hadjichrysanthou C, Cauet E, Lawrence E, Cori A.de Wolf $\mathrm{F}$, et al. How can viral dynamics models inform endpoint measures in clinical trials of therapies for acute viral infections? PLoS One. 2016.

24. Lamarca A, Clumeck N, Plettenberg A, Domingo P, Fu K, Craig $\mathrm{C}$, et al. Efficacy and safety of a once-daily fixed-dose combination of abacavir/lamivudine compared with abacavir twice daily and lamivudine once daily as separate entities in antiretroviralexperienced HIV-1-infected patients (CAL30001 Study). J Acquir Immune Defic Syndr. 2006;41:598-606. https://doi.org/10.1097/ 01.qai.0000214821.33905.5c.

25. Yusuf U, Hale GA, Carr J, Gu Z, Benaim E, Woodard P, et al. Cidofovir for the treatment of adenoviral infection in pediatric hematopoietic stem cell transplant patients. Transplantation. 2006;81:1398-404. https://doi.org/10.1097/01.tp.0000209195. $95115.8 \mathrm{e}$.

26. Heemskerk B, Lankester AC, van Vreeswijk T, Beersma MF, Claas EC, Veltrop-Duits LA, et al. Immune reconstitution and clearance of human adenovirus viremia in pediatric stem-cell recipients. J Infect Dis. 2005;191:520-30. https://doi.org/10.1086/ 427513.

27. Ljungman P, Ribaud P, Eyrich M, Matthes-Martin S, Einsele H, Bleakley $\mathrm{M}$, et al. Cidofovir for adenovirus infections after allogeneic hematopoietic stem cell transplantation: a survey by the Infectious Diseases Working Party of the European Group for Blood and Marrow Transplantation. Bone Marrow Transplant. 2003;31:481-6. https://doi.org/10.1038/sj.bmt.1703798. 